Budzyńska Julia, Leśniewska Magdalena, Kozioł Ilona, Milanowska Joanna. The impact of the COVID-19 pandemic on mental health. Journal of Education, Health and Sport. 2021;11(8):44-54. eISSN 2391-8306. DOI http://dx.doi.org/10.12775/JEHS.2021.11.08.005

https://apcz.umk.pl/czasopisma/index.php/JEHS/article/view/JEHS.2021.11.08.005

https://zenodo.org/record/5177212

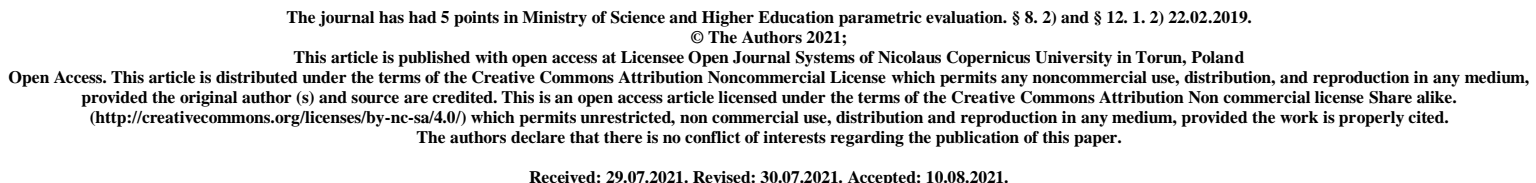

Received: 29.07.2021. Revised: 30.07.2021. Accepted: 10.08.2021.

\title{
The impact of the COVID-19 pandemic on mental health
}

\author{
Julia Budzyńska ${ }^{1}$, Magdalena Leśniewska ${ }^{1}$, Ilona Koziol ${ }^{1}$, Joanna Milanowska ${ }^{2}$
}

1. Student Scientific Circle on Department of Applied Psychology, Medical University of Lublin

2. Department of Applied Psychology, Medical University of Lublin

\begin{abstract}
Background: The outbreak of a coronavirus pandemic in 2019 posed a serious threat to the global population. Coronavirus Disease 2019 (COVID-19) has not only been a public health emergency, but has also affected mental health worldwide. Patients were exposed to a number of significant stressors during COVID-19 infection by which it appears that it may have had a major impact on mental health. Little is known about changes in levels of psychological affect, stress, anxiety and depression during this pandemic.

Aim of the study: The purpose of this study was to analyze the most recent available literature on the association of pandemic COVID-19 with psychiatric disorders occurring both as a result of past infection and in response to stress associated with the new situation of a global epidemic of Sars-CoV-2 virus.

Results: The psychological effects of the epidemic are clearly noticeable. The study identified groups particularly vulnerable to developing symptoms of anxiety, depression and post-traumatic stress disorder. Risk factors were also identified in people working in health care as having direct contact with the effects of infection with the new virus.

Summary and conclusions: Key elements of preventing psychological impact are the creation of a mental health organization. Another important task for governments is to create the conditions for economic security, as financial problems or job losses can also be the cause of mental crises.
\end{abstract}

Key Words: COVID-19 pandemic; psychological disorders; 


\section{Introduction}

A new infection caused by the new SARS-CoV-2 virus was identified in late 2019, affecting virtually all countries. Due to its very rapid spread worldwide, it posed a serious threat to the health of the general population and also posed a challenge to health care systems. The lack of knowledge about the course and prognosis of the disease and, in addition, the lack of medical and protective equipment led to the imposition of measures restricting individual freedom. Additionally, mounting financial losses have contributed to widespread emotional stress [1,2,3]. Coronavirus Disease 2019 (COVID-19) has not only been a public health emergency, but has also impacted mental health around the world, as evidenced by, for example panic buying around the world as the number of cases increases [4]. Coronaviruses may also cause psychopathological sequelae through direct viral infection of the central nervous system (CNS) or indirectly through the immune response, as observed after previous coronavirus outbreaks $[5,6]$.

Immune system disorders can significantly influence the development of psychopathology by increasing the psychological stress associated with surviving a potentially fatal disease and the accompanying stress-related inflammation. Patients were exposed to a number of significant stressors during COVID-19 infection e.g. fear of illness, uncertainty about the future, stigma, traumatic memories of severe illness and social isolation [6,7]. Little is known about changes in levels of psychological affect, stress, anxiety and depression during this pandemic [4].

\section{Aim of the study}

The purpose of this study was to analyze scientific reports on the development of psychopathology in individuals following SARS CoV-2 infection. For this purpose, the available literature was reviewed based on PubMed and Google Scholar databases. Search results were narrowed to free full text articles from the last two years - 2020 and 2021. The inclusion criteria were: discussion of the topic of mental disorders related to the past COVID19 and also related to the new situation - a global pandemic occurring in both the general population and health care workers.

\section{Tools used to measure mental health status of patients after COVID-19}

It seems that an appropriate tool dedicated to the assessment of mental health status in patients after COVID-19 has not yet been developed. One study used the National University of Singapore COVID-19 questionnaire to assess psychometric characteristics in patients in the early stages of the COVID-19 epidemic. The National University of Singapore COVID19 questionnaire consisted of five sections including questions on; demographics, physical symptoms in the past 14 days, history of contact with a patient with COVID-19 in the past 14 days, knowledge and concerns about COVID-19, and preventive measures against COVID19 in the past 14 days.

The psychological impact of COVID-19 was measured using the Impact of Event Scale-Revised (IES-R). The IES-R measures symptoms of PTSD in individuals, following a traumatic event. Respondents' mental health status was also measured using the Depression, Anxiety and Stress Scale (DASS-21). 
DASS-21 is based on a tripartite model of psychopathology that includes a general construct of distress with distinct features. IES-R and DASS were frequently used in studies related to the COVID-19 outbreak $[3,4,8,9,10]$.

In a study of 402 COVID-19 survivors, the psychopathology present was further measured using the PTSD Checklist for DSM-5 (PCL-5), Zung Depression Self-Assessment Scale (ZSDS), Beck 13-item Depression Inventory (BDI-13), State-Trait Anxiety InventoryY (STAI-Y), Medical Outcomes Study Sleep Scale (MOS-SS), Women's Health Initiative Insomnia Rating Scale (WHIIRS), and Obsessive-Compulsive Inventory (OCI) [6]. By researchers Frederikus A Klok and Gudula JAM Boon was proposed "the post COVID-19 functional status scale (PCFS)" can be assessed after hospital discharge to monitor immediate recovery and after 6 months to assess functional sequelae. This scale is intended to serve as an additional measure of the ultimate consequences of COVID-19 on the functional status of the body [11].

\section{Psychiatric symptoms in individuals following SARS CoV-2 infection}

In the study, COVID-19 survivors had high rates of PTSD, depression, anxiety, and insomnia after one month of follow-up following inpatient treatment [6].

Through social isolation, uncertainty, physical discomfort, medication side effects, fear of transmitting the virus to others, and negative social media messages, patients with COVID-19 may experience loneliness, anger, anxiety, depression, insomnia, an posttraumatic stress disorder, these for which may impair patients' social and occupational functioning and quality of life. The rapid transmission of COVID-19 and the significant publicity of the outbreak situation on social media and social discrimination against COVID19 patients may result in a higher prevalence of lowered self-esteem, post-traumatic stress symptoms associated with COVID-19 [12].

Critical illness survivors are at risk for permanent mental impairment after discharge from the hospital. Most patients with severe acute respiratory distress syndrome, which is the severe form of COVID-19 disease, show impairment in memory, attention, concentration, or speed of information processing [13].

Preliminary data suggest that patients with COVID-19 may also experience delirium, depression, anxiety, and insomnia [5,6]. Confusion and delirium are thought to be common features in the acute phase, while to date there is no data on psychopathology in the postdisease phase. Increased depression by SARSCoV-2 infection also included more frequent suicidal thoughts and suicide planning $[6,14]$.

During the initial outbreak, $15.04 \%, 9.42 \%$ and $5.62 \%$ of the respondents reported one, two or three somatic symptoms respectively. The presence of somatic symptoms has led researchers to consider the psychoneuroimmunological (PNI) effects of COVID-19 on the human body. COVID-19 can cause acute respiratory syndrome, which leads to the release of proinflammatory cytokines including interleukin (IL)-1 $\beta$ an dIL-6 from the airways. There is evidence that levels of these cytokines are elevated in major depressive disorder [4].

In a study by Eve Garrigues, Paul Janvier et al. results were obtained for the most commonly reported symptoms after COVID-19 after an average of 110.9 days, which were fatigue (55\%), dyspnea (42\%), memory loss (34\%), concentration and sleep disturbances (28\% and $30.8 \%$, respectively) [15]. 
Other studies have found that PTSS can occur in up to $96.2 \%$ of 714 hospitalized, stable patients and have shown the prevalence of depression $(29.2 \%)$ was elevated $(p=0.016)$ among the 57 patients cured with COVID-19 compared with quarantine participants $(9.8 \%)$, whereas there was no difference in anxiety $(\mathrm{P}=0.154)[12,16]$.

\section{Increased risk factors for psychiatric disorders after SARS CoV-2}

There are many risk factors for mental illness after COVID-19, especially an increased likelihood of depressive and anxiety symptoms. These include female gender, patients with a history of psychiatric symptoms, and reduced self-esteem. However, the pandemic adds the aspect of quarantine and isolation, which is also a risk factor with a psychological impact, in addition, concern for the health of family, friends and acquaintances. As the number of identified risk factors increases, the number of patients needing specialist care may increase [17].

It was proven that risk factors associated with mental disorders included having a junior high school education or less, being a corporate employee, and having PTSD symptoms. In studies, compared to women, men scored significantly higher on the GHQ-12 and PTSD scales. The difference may be due to different coping styles and social roles. Women were more likely to exhibit PTSD symptoms during the COVID-19 outbreak. Regarding education level factors, those with lower education were more likely to exhibit PTSD symptoms and psychological distress. Patients with a junior high school education or less who were screened had higher PTSD and GHQ-12 scores than other groups, which may be because higher levels of education may improve understanding of the problems associated with post-traumatic stress disorder and mental distress, leading to taking positive action to prevent the onset of related symptoms and increase confidence in mental health recovery [18].

Patients' past medical history including psychiatric history and substance abuse have been associated with an increased risk of depression and anxiety in patients after COVID-19. However, there is little knowledge about the effects of COVID-19 on patients with preexisting psychiatric disorders. Just as knowledge of the impact of previous pandemics/epidemics on this patient group is also limited [17].

Marital status was also included among the risk factors. Post-hoc LSD comparisons revealed that divorced/widowed participants had significantly higher PTSD and GHQ-12 scores than single and married/cohabiting individuals. This disparity may be due to financial losses of the family, lack of support connected with negative emotions or depressive symptoms, and lack of contact and support among immediate family members.

Studies show that among occupational groups, business groups have an increased risk of psychiatric symptoms after contracting COVID-19. They showed significantly higher PTSD scores. The problem also applies to company employees, who may be prone to psychological distress because they were forced to change their working style during the COVID-19 occurrence. Because of such a sudden change in working style, they may have difficulty adjusting to a new situation in such a short period of time, which may cause additional psychological pressure. 
Lack of coping with the situation, focusing on avoidant coping is a risk factor for posttraumatic stress symptoms, which is additionally a strong predictor of psychological distress. The risk factors presented can lead to the development and increased frequency of psychological problems, such as depression and anxiety [18].

\section{Impact of SARS CoV-2 virus infection on mental health in children and adolescents}

COVID-19 infection should be considered a risk factor for mental health disorders in children an available study of parents of children hospitalized during the epidemic found significantly higher scores for anxiety, depression, and dream anxiety compared to parents of children hospitalized during the non-COVID-19 period (all $\mathrm{p}<0.001$ ) [19]. One study showed that the prolonged blockade imposed by the Chinese government had several adverse effects on mental health, particularly among respondents aged 12 - 21.4 years. This age group consisted mainly of students affected by prolonged school closures, requiring online educational support and uncertainty about exams and matriculation conditions. On the other hand, survey respondents who were parents of children under the age of 16 were not associated with higher IES-R or DASS-21 scores. This finding is consistent with an emerging pattern of resilience to the severe effects of COVID-19 in children, and parents were less concerned as a result $[4,20]$.

One of the main reasons for this is the separation in isolation of children from their parents and guardians. They reported that insomnia and separation anxiety were the most common mental health problems presented by these children. They also emphasized the importance of screening for PTSD in patients who exhibit nightmares, avoidant behaviors, heightened reactions, and other symptoms [1]. Moreover, parents of children hospitalized for SARS CoV-2 infection may develop post-traumatic stress disorder and mental health problems, which may affect their child's recovery [19].

One study suggested that respondents who were middle school students or those who had completed that degree or less had significantly higher scores on the GHQ-12. These groups may experience long-term mental health problems as they lack the capacity to cope with mental health crises. The government and relevant psychological institutions should take appropriate psychological counseling measures to help this group return to the psychological well-being impaired by COVID-19 [18].

\section{Impact of SARS CoV-2 virus infection on the mental health of health care workers.}

According to the available literature, depression/depressive symptoms, anxiety, psychological distress, and poor sleep quality have increased among health care workers since the beginning of the COVID-19 outbreak. Additionally, female gender and frontline workers, among others, are considered to be increased risk factors [21].

A study from Wuhan city, the original outbreak of SARS CoV-2 infection, shows that psychological stress among medical personnel occurred gradually: fear and anxiety appeared earliest and decreased in the early stages of the epidemic. In contrast, depression or psychophysiological symptoms and post-traumatic stress symptoms appeared later and have longer effects. The main causes of trauma were isolation, working in high-risk jobs and contact with infected people. This could have affected medical and nursing staff working with infected people, leading to mental health problems [22]. 
It can be extremely difficult for health care professionals to deal with frightened, uncooperative, panic-stricken and stigmatized patients with COVID-19. This can generate apathy and withdrawal among clinicians. Maunder et al. showed that caring for sick colleagues during a pandemic can increase hospital staff anxiety about their competence and skills, making them more psychologically vulnerable. On the contrary, "non-essential" health workers (who are not involved in the direct care of COVID-19 patients and therefore must remain at home indefinitely during the blockades) may feel a sense of isolation and worthlessness regarding their inability to effectively contribute to the current crisis. Individuals who have hospital duties in day care and need to return home are more likely to develop a profound fear of disease transmission to their own family members, especially if there are older adults with pre-existing chronic illness who are at much higher risk for serious and adverse outcomes. In addition, the competence and mental health of newly hired employees should be carefully monitored [1].

The most common physical symptoms reported by health care workers surveyed by Nicholas W. S. Chewa et al. were headache $(289,31.9 \%)$, sore throat $(304,33.6 \%)$, anxiety $(242,26.7 \%)$, drowsiness $(241,26.6 \%)$ and insomnia $(190,21.0 \%) .302$ (33,3\%) respondents did not report any symptoms in the preceding 1 month from the date of filling in the questionnaire, $115(12.7 \%)$ reported one symptom, $113(12.5 \%)$ reported two, $73(8.1 \%)$ reported three, and 303 (33. 4\%) reported more than four symptoms. The symptoms were of mild severity. As far as the psychological symptoms reported by the study group are concerned, anxiety was found in $142(15.7 \%)$, depression in $96(10.6 \%)$ and stress in 47(5. $2 \%$ ) study participants. For $50 \%$ of them (48), depression was rated as moderate or very severe. In the 142 patients who were diagnosed with anxiety, 55. 6\% had moderate to severe anxiety. $67(7,4 \%)$ tested positive for PTSD clinically, of which 34 had moderate to severe levels of psychological distress. The same study found that physical symptoms were more common in people with comorbidities.[3] Another study reveals that $10.8 \%$ of respondents struggle with PTSD symptoms, with concurrent low prevalence of anxiety (3. 8\%), depression (3. $7 \%)$, stress $(1.5 \%)$ and insomnia $(2.3 \%)[23,24]$.

\section{Therapies used to treat trauma versus COVID-19}

The presence of PTSD symptoms can be observed among COVID-19 patients. One example of treatment for these symptoms is trauma-focused cognitive-behavioral therapy (TF-CBT). TF-CBT can be modified for COVID-19 pandemic with emphasis on education about the psychological impact of COVID-19 pandemic, psychosocial skill development to optimize emotional and behavioral adjustment during quarantine and confinement, trauma narrative, home-based relaxation techniques and stress management skills with online guidance, grief therapy to cope with the potential loss of family members or friends, who died from COVID-19, increased safety and precautions to reduce the risk of contracting COVID-19. Implementing TF-CBT during the COVID-19 pandemic requires healthcare organizations to develop online preparedness and additionally engage counselors, psychologists, physicians, insurers and the public to accept new models of mental health services [4]. 


\section{Summary and conclusion}

The available literature suggests that COVID-19 survivors may have a higher incidence of psychiatric conditions, including mood disorders, PTSD, and insomnia. Available data indicate that confusion and delirium are common features in the acute phase, whereas to date there is no data on psychopathology in the post-disease phase [6,21]. There is a relationship between the presence of somatic and psychiatric symptoms. It is recommended that psychological support should be provided to medical staff when physical symptoms are present and when infection has been ruled out. There is greater anxiety associated with COVID-19 in non-medical health care workers [3,25]. Symptoms of moderate to severe severity are more common in the general population compared to the group of healthcare workers surveyed. However, physical symptoms such as headache or sore throat were more common in health care than the general population [3]. The mental problems associated with COVID-19 may not only be related to psychological stress and trauma, but may be the result of damage to brain structures following systemic inflammation or hypoxia [14,26,27].

Studies show that most patients requiring hospitalization for COVID-19 have persistent symptoms, even 110 days after hospital discharge, especially fatigue and dyspnea. These findings highlight the need for long-term follow-up of these patients and rehabilitation programs [15]. Because of the impact of COVID-19 infection on mental health, the psychopathology of COVID-19 survivors should be evaluated to diagnose and treat emerging psychiatric conditions and to monitor these changes over time [6]. The available literature suggests that middle school students and those with less education have an increased risk of psychiatric symptoms following COVID-19. For this reason, the government and relevant psychological institutions should take appropriate psychological counseling measures to help this group recover from consequences of Sars-CoV-2 infection [18]. The important need for social support and early psychological and psychiatric help for children, parents and carers experiencing anxiety during a pandemic was noted [1]. The dissemination of large amounts of health information through the media was associated with higher levels of anxiety and depression. Such observations may influence actions taken by government and health authorities around the world in modifying current methods of raising public awareness and communicating the course of the pandemic [4].

Most assistance should be prepared for vulnerable groups: women, people under 40, university students and people with psychiatric illnesses and chronic mental health problems. Psychological support may be provided online via telepresence due to the limited availability of face-to-face consultations. It is also very important to take care of one's own mental health through a healthy lifestyle (physical activity, balanced diet) as well as various forms of taking care of one's own mental well-being (phone and video conversations with loved ones). Another important element is access to reliable information on the pandemic as well as on ways of limiting virus transmission [28]. On the other hand, overexposure to current COVID19 threat statistics should be avoided.

Aspects of employment have a significant impact on mental health. Anxiety, depression, PTSD, suicidal thoughts, sleep disorders, and drug and alcohol addiction are more likely to affect health care workers, especially those working on the front lines and those in contact with the public such as law enforcement personnel, among others. 
This is associated with a sense of fear of being a vector of the virus, infection of family members, and stigmatization [24].

Some groups have experienced social discrimination due to the COVID-19 outbreak. As a result, WHO and governments of all countries should take action to reduce the potential for discrimination on the basis of COVID-19. It should also ensure the availability of basic services and goods, and strengthen financial security. These measures are necessary to prevent the psychological impact of the current pandemic [4].

There is a need to establish mental health organizations specific to future pandemics with branches in multiple countries and in individual health care institutions to conduct research, provide mental health care, and organize an awareness program at both the individual and community levels. Structured websites and toll-free helplines can be launched to alleviate mental stress among the general public due to the ongoing pandemic. Social media can be used in a good sense to educate people about the dynamics of transmission, symptoms of the disease, and when accurate medical consultations are needed. To protect social media from devaluation, strict government regulations and laws on fake news, social media gossip and misinformation must be implemented [29]. 


\section{References}

1. Marques de Miranda D, da Silva Athanasio B, Sena Oliveira AC, Simoes-E-Silva AC. How is COVID-19 pandemic impacting mental health of children and adolescents? Int J Disaster Risk Reduct. 2020 Dec;51:101845. doi: 10.1016/j.ijdrr.2020.101845. Epub 2020 Sep 10.

2. Pfefferbaum B, North CS. Mental Health and the Covid-19 Pandemic. N Engl J Med. 2020 Aug 6;383(6):510-512. doi: 10.1056/NEJMp2008017. Epub 2020 Apr 13

3. Chew, N. W. S., Lee, G. K. H., Tan, B. Y. Q., Jing, M., Goh, Y., Ngiam, N. J. H., Yeo, L. L. L., Ahmad, A., Ahmed Khan, F., Napolean Shanmugam, G., Sharma, A. K., Komalkumar, R. N., Meenakshi, P. V., Shah, K., Patel, B., Chan, B. P. L., Sunny, S., Chandra, B., Ong, J. J. Y., ... Sharma, V. K. (2020). A multinational, multicentre study on the psychological outcomes and associated physical symptoms amongst healthcare workers during COVID-19 outbreak. Brain, Behavior, and Immunity, 88. https://doi.org/10.1016/j.bbi.2020.04.049

4. Wang, C., Pan, R., Wan, X., Tan, Y., Xu, L., McIntyre, R. S., Choo, F. N., Tran, B., Ho, R., Sharma, V. K., \& Ho, C. (2020). A longitudinal study on the mental health of general population during the COVID-19 epidemic in China. Brain, Behavior, and Immunity, 87. https://doi.org/10.1016/j.bbi.2020.04.028

5. Wu Y, Xu X, Chen Z, Duan J, Hashimoto K, Yang L, Liu C, Yang C. Nervous system involvement after infection with COVID-19 and other coronaviruses. Brain Behav Immun. 2020 Jul;87:18-22. doi: 10.1016/j.bbi.2020.03.031. Epub 2020 Mar 30.

6. Mazza MG, De Lorenzo R, Conte C, Poletti S, Vai B, Bollettini I, Melloni EMT, Furlan R, Ciceri F, Rovere-Querini P; COVID-19 BioB Outpatient Clinic Study group, Benedetti F. Anxiety and depression in COVID-19 survivors: Role of inflammatory and clinical predictors. Brain Behav Immun. 2020 Oct;89:594-600. doi: 10.1016/j.bbi.2020.07.037. Epub 2020 Jul 30.

7. Carvalho PMM, Moreira MM, de Oliveira MNA, Landim JMM, Neto MLR. The psychiatric impact of the novel coronavirus outbreak. Psychiatry Res. 2020 Feb 28;286:112902. doi: 10.1016/j.psychres.2020.112902. Epub ahead of print.

8. Wang C, Pan R, Wan X, Tan Y, Xu L, Ho CS, Ho RC. Immediate Psychological Responses and Associated Factors during the Initial Stage of the 2019 Coronavirus Disease (COVID-19) Epidemic among the General Population in China. Int J Environ Res Public Health. 2020 Mar 6;17(5):1729. doi: 10.3390/ijerph17051729.

9. Christianson S, Marren J. The Impact of Event Scale - Revised (IES-R). Medsurg Nurs. 2012 Sep-Oct;21(5):321-2.

10. Hosey MM, Bienvenu OJ, Dinglas VD, Turnbull AE, Parker AM, Hopkins RO, Neufeld KJ, Needham DM. The IES-R remains a core outcome measure for PTSD in critical illness survivorship research. Crit Care. 2019 Nov 19;23(1):362. doi: 10.1186/s13054-019-2630-3.

11. Klok FA, Boon GJAM, Barco S, Endres M, Geelhoed JJM, Knauss S, Rezek SA, Spruit MA, Vehreschild J, Siegerink B. The Post-COVID-19 Functional Status scale: a tool to measure functional status over time after COVID-19. Eur Respir J. $2020 \mathrm{Jul}$ 2;56(1):2001494. doi: 10.1183/13993003.01494-2020. 
12. Bo HX, Li W, Yang Y, Wang Y, Zhang Q, Cheung T, Wu X, Xiang YT. Posttraumatic stress symptoms and attitude toward crisis mental health services among clinically stable patients with COVID-19 in China. Psychol Med. 2021 Apr;51(6):1052-1053. doi: 10.1017/S0033291720000999. Epub 2020 Mar 27.

13. Rogers JP, Chesney E, Oliver D, Pollak TA, McGuire P, Fusar-Poli P, Zandi MS, Lewis G, David AS. Psychiatric and neuropsychiatric presentations associated with severe coronavirus infections: a systematic review and meta-analysis with comparison to the COVID-19 pandemic. Lancet Psychiatry. 2020 Jul;7(7):611-627. doi: 10.1016/S2215-0366(20)30203-0. Epub 2020 May 18.

14. Sher L. Post-COVID syndrome and suicide risk. QJM. 2021 Apr 27;114(2):95-98. doi: 10.1093/qjmed/hcab007.

15. Garrigues, E., Janvier, P., Kherabi, Y., Le Bot, A., Hamon, A., Gouze, H., Doucet, L., Berkani, S., Oliosi, E., Mallart, E., Corre, F., Zarrouk, V., Moyer, J. D., Galy, A., Honsel, V., Fantin, B., \& Nguyen, Y. (2020). Post-discharge persistent symptoms and health-related quality of life after hospitalization for COVID-19. Journal of Infection, 81(6). https://doi.org/10.1016/j.jinf.2020.08.02

16. Zhang J, Lu H, Zeng H, Zhang S, Du Q, Jiang T, Du B. The differential psychological distress of populations affected by the COVID-19 pandemic. Brain Behav Immun. 2020 Jul;87:49-50. doi: 10.1016/j.bbi.2020.04.031. Epub 2020 Apr 15.

17. Vindegaard N, Benros ME. COVID-19 pandemic and mental health consequences: Systematic review of the current evidence. Brain Behav Immun. 2020 Oct;89:531542. doi: 10.1016/j.bbi.2020.05.048. Epub 2020 May 30.

18. Liang L, Ren H, Cao R, Hu Y, Qin Z, Li C, Mei S. The Effect of COVID-19 on Youth Mental Health. Psychiatr Q. 2020 Sep;91(3):841-852. doi: 10.1007/s11126020-09744-3.

19. Yuan R, Xu QH, Xia CC, Lou CY, Xie Z, Ge QM, Shao Y. Psychological status of parents of hospitalized children during the COVID-19 epidemic in China. Psychiatry Res. 2020 Jun;288:112953. doi: 10.1016/j.psychres.2020.112953. Epub 2020 Apr 13.

20. Sominsky L, Walker DW, Spencer SJ. One size does not fit all - Patterns of vulnerability and resilience in the COVID-19 pandemic and why heterogeneity of disease matters. Brain Behav Immun. 2020 Jul;87:1-3. doi: 10.1016/j.bbi.2020.03.016. Epub 2020 Mar 20.

21. Vindegaard N, Benros ME. COVID-19 pandemic and mental health consequences: Systematic review of the current evidence. Brain Behav Immun. 2020 Oct;89:531542. doi: 10.1016/j.bbi.2020.05.048. Epub 2020 May 30.

22. Lijun Kang, Simeng Ma, Min Chen, Jun Yang, Ying Wang, Ruiting Li, Lihua Yao, Hanping Bai, Zhongxiang Cai, Bing Xiang Yang, Shaohua Hu, Kerang Zhang, Gaohua Wang, Ci Ma, Zhongchun Liu. Impact on mental health and perceptions of psychological care among medical and nursing staff in Wuhan during the 2019 novel coronavirus disease outbreak: Across-sectional study. Brain, Behavior, and Immunity, Volume 87, 2020, Pages 11-17, ISSN 0889-1591. doi: 10.1016/j.bbi.2020.03.028.

23. Tan W, Hao F, McIntyre RS, Jiang L, Jiang X, Zhang L, Zhao X, Zou Y, Hu Y, Luo $\mathrm{X}$, Zhang Z, Lai A, Ho R, Tran B, Ho C, Tam W. Is returning to work during the 
COVID-19 pandemic stressful? A study on immediate mental health status and psychoneuroimmunity prevention measures of Chinese workforce. Brain Behav Immun. 2020 Jul;87:84-92. doi: 10.1016/j.bbi.2020.04.055. Epub 2020 Apr 23.

24. Giorgi G, Lecca LI, Alessio F, Finstad GL, Bondanini G, Lulli LG, Arcangeli G, Mucci N. COVID-19-Related Mental Health Effects in the Workplace: A Narrative Review. Int J Environ Res Public Health. 2020 Oct 27;17(21):7857. doi: 10.3390/ijerph17217857.

25. Tan BYQ, Chew NWS, Lee GKH, Jing M, Goh Y, Yeo LLL, Zhang K, Chin HK, Ahmad A, Khan FA, Shanmugam GN, Chan BPL, Sunny S, Chandra B, Ong JJY, Paliwal PR, Wong LYH, Sagayanathan R, Chen JT, Ng AYY, Teoh HL, Ho CS, Ho RC, Sharma VK. Psychological Impact of the COVID-19 Pandemic on Health Care Workers in Singapore. Ann Intern Med. 2020 Aug 18;173(4):317-320. doi: 10.7326/M20-1083. Epub 2020 Apr 6.

26. Steardo L Jr, Steardo L, Verkhratsky A. Psychiatric face of COVID-19. Transl Psychiatry. 2020 Jul 30;10(1):261. doi: 10.1038/s41398-020-00949-5.

27. Xiong J, Lipsitz O, Nasri F, Lui LMW, Gill H, Phan L, Chen-Li D, Iacobucci M, Ho R, Majeed A, McIntyre RS. Impact of COVID-19 pandemic on mental health in the general population: A systematic review. J Affect Disord. 2020 Dec 1;277:55-64. doi: 10.1016/j.jad.2020.08.001. Epub 2020 Aug 8.

28. Meyer JH, Cervenka S, Kim MJ, Kreisl WC, Henter ID, Innis RB. Neuroinflammation in psychiatric disorders: PET imaging and promising new targets. Lancet Psychiatry. 2020 Dec;7(12):1064-1074. doi: 10.1016/S2215-0366(20)30255-8. Epub 2020 Oct 21.

29. Dubey S, Biswas P, Ghosh R, Chatterjee S, Dubey MJ, Chatterjee S, Lahiri D, Lavie CJ. Psychosocial impact of COVID-19. Diabetes Metab Syndr. 2020 SepOct;14(5):779-788. doi: 10.1016/j.dsx.2020.05.035. Epub 2020 May 27 